\title{
Avaliação da morfometria renal através do método ultrassonográfico de fêmeas caprinas (Capra hircus) normais da raça Saanen ${ }^{1}$
}

\author{
Rodolfo S. Rossi² ${ }^{2}$ Pedro P. Bombonato ${ }^{3}$, Fernanda M. Piva² e Lílian Gregory²*
}

\begin{abstract}
Rossi R.S., Bombonato P.P., Piva F.M. \& Gregory L. 2012. [Renal morphometric evaluation by ultrasonographic techniques in normal female Saanen goats (Capra hircus).] Avaliação da morfometria renal através do método ultrassonográfico de fêmeas caprinas (Capra hircus) normais da raça Saanen. Pesquisa Veterinária Brasileira 32(2):165173. Departamento de Clínica Médica, Faculdade de Medicina Veterinária e Zootecnia, Universidade de São Paulo, Av. Prof. Dr. Orlando Marques de Paiva 87, São Paulo, SP 05508270, Brazil. E-mail: lgregory@usp.br

The veterinary ultrasonographic techniques have increased in the veterinary doctor's daily work, therefore training and knowing how to use ultrasound equipment have become needful to the animals healthcare professionals. It's important remember that ultrasound equipment makes possible getting valuable pathological data from animals' health. In this background, the renal ultrasonographic test has gotten an important place and, nowadays, it is possible measure with accuracy the caprine renal morphometrics parameters (Capra hircus). Through ultrasonographic techniques, it has done a stocktaking with three groups of normal goats: older than 18 months ( $46.3 \pm 17.4$ months), younger than 6 months $(3.0 \pm 1.0$ months) and age between 6 and 18 months (9.0 4.3 months), totalizing 30 samples of the female Saanen kind. In longitudinal section of the kidneys, measured the length and breadth and, in transverse section, measured the height (or thickness). The values obtained calculated the renal, cortical and medullary volumes, besides corticomedullary correlation. The renal length of the groups younger than 6 months, age between 6 and 18 months and older than 18 months had mean and standard deviation of $4.20 \pm 0.36 \mathrm{~cm} ; 5.56 \pm 0,40 \mathrm{~cm}$; and, $6.77 \pm 0.64 \mathrm{~cm}$, respectively. To the renal volume theses groups had mean and standard deviation of $17.02 \pm 3.99 \mathrm{~cm}^{3} ; 19.99 \pm 5.86 \mathrm{~cm}^{3}$; and, $41.23 \pm 13.05 \mathrm{~cm}^{3}$. It was compared the metrical equivalence of the mean between the two kidneys, to the group with age between 6 and 18 months the parameters showed difference were renal length, renal volume and cortical volume, in other hand to the group older than 18 months it was renal length and medullar length. Comparing different groups was noted that only the left medullar length had equivalent mean in all the groups, it means, the group younger than 6 months, older than 18 months and from 6 to 18 months of age. The results showed direct and positive correlation between corporal weight and age with the linear and volumetric parameters The left corticomedullary correlation was the only of its kind to exhibit significant correlation with the weight $(r=-0.365$; $P$ value 0.047$)$. The parameters with significant correlation had the regression analysis done, obtained the better line of adjust of the variables.
\end{abstract}

INDEX TERMS: Ultrasound, goats, kidney, renal function, clinics, ruminant

\footnotetext{
${ }^{1}$ Recebido em 6 de abril de 2011.

Aceito para publicação em 20 de outubro de 2011.

${ }^{2}$ Departamento de Clínica Médica (VCM), Faculdade de Medicina Veterinária e Zootecnia (FMVZ), Universidade de São Paulo (USP), Av. Prof. Dr. Orlando Marques de Paiva 87, Cidade Universitária, São Paulo, SP 05508270, Brasil. *Autor para correspondência: Igregory@usp.br

${ }^{3}$ Departamento de Cirurgia (VCI) ), FMVZ-USP, Av. Prof. Dr. Orlando Marques de Paiva 87, São Paulo, SP 05508-270.
}

RESUMO.- A utilização de técnicas ultrassonográficas na área de Medicina Veterinária está cada vez mais presente e a capacitação nesta área tornou-se indispensável para o clínico, auxiliando-o na obtenção de informações rápidas e valiosas das afecções patológicas que podem acometer os animais. 0 exame ultrassonográfico renal revelou-se de grande importância neste âmbito, com o objetivo de avaliar 
e mensurar os parâmetros morfométricos renais normais de fêmeas caprinas (Capra hircus). Por meio de técnicas ultrassonográficas procedeu-se o estudo de 30 fêmeas da raça Saanen, divididas em três grupos: fêmeas com idade inferior a 6 meses $(3,0 \pm 1,0$ meses), de $6-18$ meses $(9,0 \pm 4,3$ meses) e com idade superior a 18 meses $(46,3 \pm 17,4$ meses). Realizaram-se imagens dos rins, em secções longitudinais, medidas de comprimento e largura e, em secções transversais, medidas de altura (ou espessura). Com estes valores calcularam-se volumes renais, corticais e medulares, além da relação cortico-medular. Com relação ao comprimento renal os grupos com idade inferior a 6 meses, de 6-18 meses e com idade superior a 18 meses obtiveram média e desvio padrão de $4,20 \pm 0,36 \mathrm{~cm}, 5,56 \pm 0,40 \mathrm{~cm}$ e $6,77 \pm 0,64 \mathrm{~cm}$, respectivamente. Tratando-se do volume renal, estes grupos apresentaram média e desvio padrão de $17,02 \pm 3,99 \mathrm{~cm}^{3}$, $19,99 \pm 5,86 \mathrm{~cm}^{3} ; \mathrm{e}, 41,23 \pm 13,05 \mathrm{~cm}^{3}$. Comparou-se a equivalência métrica das médias entre os dois rins de forma que os parâmetros volumétricos e lineares renais com diferença entre si são comprimento renal, volume renal e volume cortical para o grupo de fêmeas com idade de 6-18 meses, e comprimento renal e comprimento medular para o grupo com idade superior a 18 meses. Entre diferentes grupos observou-se que somente o comprimento medular esquerdo apresentou média equivalente em todos os grupos, ou seja, entre o grupo com idade inferior a 6 meses e o grupo com idade de 6-18 meses e, entre este último e o grupo com idade superior a 18 meses. Os resultados mostraram correlações diretas e positivas entre peso corporal e idade com os parâmetros lineares e volumétricos, a relação cortico-medular esquerda foi a única que apresentou correlação significativa com o peso $(r=-0,365 ; P=0,047)$. Para aqueles parâmetros que apresentaram correlação significativa foi realizada análise de regressão, obtendo-se a linha de melhor ajuste das variáveis.

TERMOS DE INDEXAÇÃO: Ultrassom, caprinos, rim, função renal, clínica, ruminantes.

\section{INTRODUÇÃO}

Diante da grande importância dos rins frente à manutenção da homeostasia do organismo, o estudo para determinação ou avaliação da variabilidade dos valores métricos de referência dos parâmetros fisiológicos de animais domésticos sadios é fundamental para que o clínico veterinário possa analisar e comparar possíveis alterações decorrentes de diversas enfermidades (Coles 1986, Kaneko et al. 1997). Alguns dados podem ressaltar a importância das dimensões da função renal, por exemplo, em cães de grande porte (e animais de tamanho similar), nos quais 1.000 a 2000 litros de sangue perfundem diariamente os rins, obtendo-se 200 a 300 litros de líquido filtrado que são, posteriormente, reduzidos por reabsorção até que 1 ou 2 litros de urina remanescente sejam eliminados (Dyce et al. 2004).

Para avaliação de pacientes, o exame físico é fundamental para detectar alterações funcionais de certos órgãos ou sistemas, abrangendo dentre estes o exame renal o qual, no entanto, apresenta limitações (Coles 1986, Kaneko et al. 1997). Para auxiliar na avaliação renal o clínico pode valer-se do es- tudo ultrassonográfico, o qual é um método de auxílio diagnóstico que vem sendo utilizado frequentemente, por sua grande viabilidade e veracidade em medidas e imagens (Piera 2005). A principal vantagem do ultrassom é a sua capacidade de discriminação dos diferentes elementos anatômicos constituintes do órgão quer seja, a cápsula, córtex e medula renal, divertículos pélvicos e seio renal. Permite também a diferenciação das lesões dentro do rim, isto é, se é uma lesão focal, multifocal ou difusa (Dibartola 1996).

Além da anatomia, pode-se avaliar o volume e a área renal através da ultrassonografia. A determinação dessas medidas tem potencial importância numa grande variedade de situações clínicas. Uma obstrução ureteral ou aumento do fluido urinário podem fazer com que haja um aumento no valor de volume renal (Kealy \& McAllister 2005).

Os rins estão cobertos por uma cápsula de tecido conjuntivo que consiste em uma rede fibrosa colágena com uma pequena quantidade de fibras elásticas. A cápsula mergulha no hilo e forma o seio renal. 0 rim consiste de um córtex externo e uma medular interna. 0 córtex está pontilhado por diminutos pontos escuros, os corpúsculos do rim, cada um consistindo na origem dilatada de um túbulo renal com um tufo invaginado de capilares (glomérulos) por ele circundados. A medula projeta-se dentro da pelve renal ou cálices renais, com uma ou mais papilas. As papilas são os ápices das pirâmides renais (Dyce et al. 2004).

Nos pequenos ruminantes, a posição dos rins é a mesma dos bovinos, mas com estrutura diferente. Eles são lisos na superfície e possuem a crista renal (pirâmide ou papila renal) e a pelve como aquelas dos cães (Habel 1970). 0 rim direito localiza-se na região retroperitoneal e é circundado por tecido adiposo, sob a $13^{\text {a }}$ costela e os três primeiros processos transversos lombares. É palpável na fossa paralombar nos ruminantes vivos. A extremidade cranial ajusta-se ao processo caudado do fígado e o hilo encontra-se na face ventro-medial (Habel 1970). Já o rim esquerdo é situado ventral às $3^{a}$, $4^{\underline{a}}$ e $5^{\underline{a}}$ vértebras lombares e penduloso, quase completamente envolto pelo peritônio (Habel 1970), sendo empurrado para metade direita do abdômen pelo desenvolvimento do rúmen, processo que se intensifica quando há seu enchimento (Dyce et al. 2004).

Na ultrassonografia, áreas anatômicas individuais apresentam padrões acústicos característicos (Konde 1989). No corte transversal identificamos região cortical, região medular, divertículos, pelve renal e gordura peripélvica (Carvalho 2004).No corte longitudinal do rim, podemos identificar a cápsula renal, que produz um fino eco brilhante, quando a onda sonora incide perpendicularmente. A região cortical é mais ecogênica que a região medular porque é formada pelos glomérulos, possuindo uma quantidade maior de células que a região medular; esta última é composta pela maioria dos túbulos do sistema coletor, possuindo uma maior quantidade de fluido, o que a torna hipoecogênica. Por fim, a porção mais central hiperecogênica chamada de complexo ecogênico central corresponde à pelve e gordura peripélvica (Carvalho 2004).

Existe uma demarcação evidente entre as regiões cortical e medular, chamada de junção cortico-medular, onde se 
localizam as artérias e veias arqueadas. As linhas ecogênicas que acompanham as artérias e veias interlobares são os divertículos dorsais e ventrais, espaçados regularmente e localizados na região medular, dividindo-a em segmentos. A medula, portanto, é dividida em múltiplas secções pelos divertículos e vasos interlobares. A borda medial é identificada pela abertura oval chamada hilo, por de onde saem a veia renal e o ureter e entra a artéria renal (Carvalho 2004).

Algumas características podem dificultar o exame como o acúmulo de gordura ou ar e a interferência dos ossos, mas com o uso de técnicas ultrassonográficas apropriadas e o conhecimento das ferramentas do ultrassom podemos obter a imagem do órgão ou região desejada (Piera 2005). 0 exame dos rins deve ser realizado com cuidado e paciência, com a utilização criteriosa dos planos de corte, já que a sua visibilização incompleta pode causar erros de interpretação (Carvalho 2004).

O objetivo do trabalho foi obter por meio da ultrassonografia, em condições fisiológicas normais, os valores de referência dos parâmetros morfométricos renais em fêmeas caprinas normais (Capra hircus) da raça Saanen em grupos de animais com idade inferior a 6 meses, de 6 a 18 meses e com idade superior a 18 meses.

\section{MATERIAL E MÉTODOS}

Para a realização deste trabalho foram utilizadas 30 fêmeas previamente selecionadas da raça Saanen, divididas em três grupos: 10 fêmeas com idade inferior a 6 meses $(3,0 \pm 1,0$ meses $), 7$ fêmeas com idade entre 6 e 18 meses $(9,0 \pm 4,3$ meses) e 13 fêmeas com idade superior a 18 meses $(46,3 \pm 17,4$ meses $)$, com seus pesos mensurados. 0 limite de idade inferior e superior para cada grupo foi de 1,7 e 5,3 meses, 6,3 e 15,9 meses, e 20,2 e 74,1 meses, respectivamente. Tomou-se o cuidado de utilizar fêmeas que não estivessem prenhes ou apresentassem sinais clínicos relativos a quadros patológicos renais, para tanto foi realizado exame clínico de suas funções vitais.

Os animais foram tricotomizados na região do flanco direito, cranialmente a $12^{\mathrm{a}}$ e $13^{\mathrm{a}}$ costela, e caudalmente margeando à asa do íleo. Os exames de ultrassom foram realizados com aparelho da marca Pie Medical, modelo Falco Vet, com Modo B e com o auxílio de um transdutor trans-retal de $6 \mathrm{MHz}$, o que proporcionou boa resolução da imagem e visualização de detalhes do parênquima renal. Para reduzir as variáveis que podiam influenciar na realização das práticas ultrassonográficas, todas as mensurações foram padronizadas sendo realizadas com os animais em estação, e, em virtude da grande mobilidade do rim esquerdo, procurou-se fixá-los manualmente, o que facilitou a realização dos planos de corte. Foram obtidas imagens ultrassonográficas dos rins em planos de cortes longitudinais e transversais, para obtenção de dados relativos à topografia, contornos, arquitetura, ecogenicidade e ecotextura, e, principalmente, dos dados métricos.

As imagens obtidas através de cortes longitudinais forneceram dados relativos ao comprimento e a largura. 0 comprimento foi tomado como sendo a distância máxima entre a margem renal cranial e caudal, já a largura foi obtida mensurando-se as distâncias máximas entre as margens lateral e medial dos rins.

As imagens referentes aos cortes transversais, obtidas com a rotação de $90^{\circ}$ do transdutor trans-retal, forneceram os valores relativos à altura (ou espessura), sendo esta tomada como sendo a distância máxima entre a margem renal dorsal e ventral.

Para avaliar a relação cortico-medular pelo volume, partiu-se do princípio que rins e medulas renais assumem formas elipsói- des, assim usou-se uma fórmula conhecida e estudada por autores para obtermos os volumes desejados (Volume $=$ Comprimento $\mathrm{x}$ Altura x Largura x 0,523). 0 valor 0,523 representa uma constante, $\pi / 6$, considerando-se o rim como uma elipse. (Nyland et. al. 1989, Barr 1990, Felkai et. al. 1992, Bakker et al. 1999).

Com estas medidas foram calculados o volume renal (VR) e o volume medular (VM), chegando então ao volume da cortical (VC) pela diferença entre os dois (VC = VR-VM). Após a obtenção dos valores dos volumes medidos a relação cortico-medular (RCM) foi realizada por relação simples de VC/VM.

Com os dados morfométricos obtidos, a análise estatística foi realizada segundo Berquó et al. (1981), similarmente aos autores Barr et al. (1990), Tello \& Requeséns (1995), Sampaio \& Araújo (2001), Cunha (2005), os quais também realizaram trabalhos na área. Os parâmetros estatísticos avaliados foram medidas de tendência central, coeficientes de correlação de Pearson, teste de normalidade de Anderson-Darling, análise de variância (teste de Fisher), teste $t$ de Student e análise de regressão, com nível de significância de $\mathrm{P}<0,05$.

\section{RESULTADOS}

0 presente estudo mensurou, por meio da ultrassonografia, a morfometria renal de 30 fêmeas sadias da raça Saanen, agrupadas de acordo com a idade em três grupos. Considerando-se o peso a média geral das fêmeas de 28,4 kg apresentou expressiva variabilidade com desvio padrão de 19,1 kg. Para os grupos com idade inferior a 6 meses, de 6-18 meses e com idade superior a 18 meses obteve-se média e

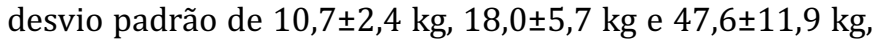
respectivamente. Os limites inferiores e superiores foram de 5,5 e 13,8 kg, 13 e 29,5 kg, e, 28,7 e 65,2 kg, respectivamente, para cada grupo.

0 teste $\mathrm{t}$ de Student realizado com base nas medidas mensuradas revelou que para o grupo de fêmeas com idade inferior a 6 meses todos os parâmetros lineares (comprimento, largura e altura, tanto renal como medular) possuem suas médias equivalentes entre os dois rins. 0 grupo com idade de 6-18 meses, também apresentou médias estatisticamente equivalentes para os parâmetros lineares, exceto quando trata-se do comprimento renal, o qual possui o comprimento esquerdo em média $0,541 \mathrm{~cm}$ superior ao comprimento renal direito. Já para o grupo com idade superior a 18 meses os parâmetros que apresentaram diferenças nas médias entre os dois rins, foram o comprimento renal e o comprimento medular, de forma que os valores são, respectivamente, em média $0,828 \mathrm{~cm}$ e $0,687 \mathrm{~cm}$ superiores para o rim esquerdo (Quadro 1).

Pelo mesmo teste ( $t$ de Student) realizado para os dados volumétricos observou-se que para os grupos com idade inferior a 6 meses e superior a 18 meses todos os parâmetros volumétricos (volumes renal, cortical e medular) e relação cortico-medular apresentaram suas médias estatisticamente equivalentes entre os dois rins. No entanto, para o grupo de fêmeas com idade de 6-18 meses constatou-se que o volume renal e o volume cortical não são equivalentes entre os rins, sendo em média o esquerdo $6,33 \mathrm{~cm}^{3} \mathrm{e}$ $5,67 \mathrm{~cm}^{3}$ superior ao direito, respectivamente (Quadro 2).

A análise, ainda com a aplicação do teste $t$ de Student, entre os grupos de fêmea scom idade inferior a 6 meses e entre 6 a 18 meses o comprimento renal esquerdo e o 
Quadro 1. Resultados estatísticos do comprimento, largura e altura, renal e medular dos rins das 30 fêmeas

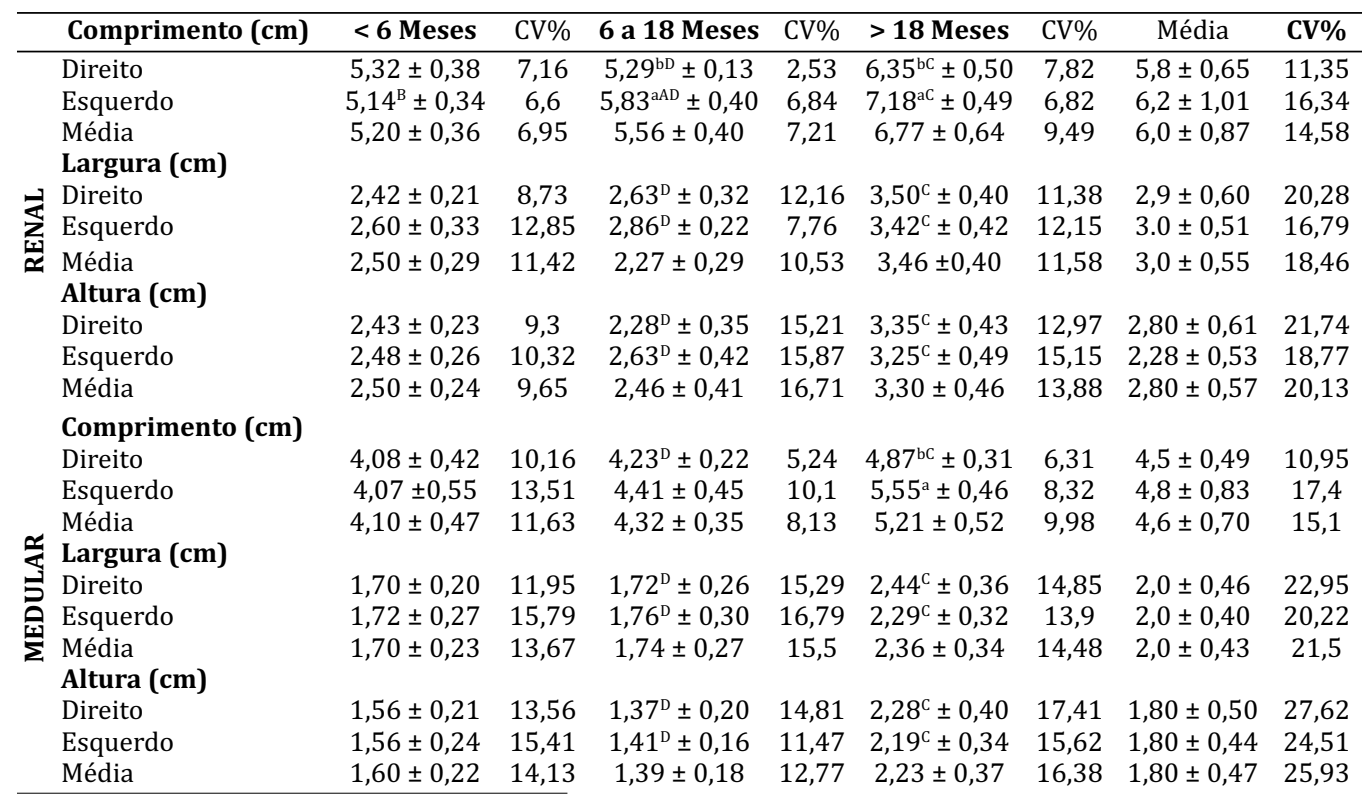

a-b,A-B,C-D Médias \pm desvio padrão na coluna que não compartilham letra minúscula e na linha letra maiúscula sobrescritas são diferentes estatisticamente pelo teste de Tuckey $(\mathrm{P}<0.05)$.

Quadro 2. Resultados estatísticos dos volumes renal, cortical e medular, e relação cortico-medular dos rins das 30 fêmeas

\begin{tabular}{|c|c|c|c|c|c|c|c|c|}
\hline Volume Renal $\left(\mathrm{cm}^{3}\right)$ & $<6$ Meses & CV\% & 6 a 18 Meses & CV\% & $>18$ Meses & CV\% & Média & CV\% \\
\hline Direito & $16,48 \pm 3,40$ & 20,51 & $16,82^{\mathrm{bD}} \pm 4,16$ & 24,71 & $39,68^{\mathrm{C}} \pm 11,72$ & 29,53 & $26,21 \pm 13,87$ & 53,02 \\
\hline Esquerdo & $17,56 \pm 4,63$ & 26,38 & $23,15^{\mathrm{aD}} \pm 5,80$ & 25,04 & $42,77^{\mathrm{C}} \pm 14,57$ & 34,06 & $29,79 \pm 15,20$ & 51,91 \\
\hline Média & $17,02 \pm 3,99$ & 23,46 & $19,99 \pm 5,86$ & 29,30 & $41,23 \pm 13,05$ & 31,65 & $28,20 \pm 14,76$ & 52,35 \\
\hline \multicolumn{9}{|l|}{ Volume Cortical (cm3) } \\
\hline Direito & $10,70 \pm 2,42$ & 22,64 & $11,54^{\mathrm{bD}} \pm 3,07$ & 26,59 & $25,21^{\mathrm{C}} \pm 9,94$ & 39,44 & $17,18 \pm 9,62$ & 56,94 \\
\hline Esquerdo & $11,62^{\mathrm{B}} \pm 3,45$ & 29,69 & $17,21^{\mathrm{aAD}} \pm 4,94$ & 28,69 & $27,97^{\mathrm{C}} \pm 10,32$ & 36,89 & $20,01 \pm 10,19$ & 51,78 \\
\hline Média & $11,16 \pm 2,94$ & 26,33 & $14,37 \pm 4,92$ & 34,26 & $26,59 \pm 10,03$ & 37,71 & $18,60 \pm 10,09$ & 54,27 \\
\hline \multicolumn{9}{|l|}{ Volume Medular (cm3) } \\
\hline Direito & $5,78 \pm 1,68$ & 29,04 & $5,28^{D} \pm 1,37$ & 25,97 & $14,48^{C} \pm 4,56$ & 31,51 & $9,43 \pm 5,39$ & 58,12 \\
\hline Esquerdo & $5,94 \pm 2,31$ & 38,87 & $5,95^{\mathrm{D}} \pm 2,21$ & 37,22 & $14,80^{C} \pm 4,51$ & 30,46 & $9,78 \pm 5,47$ & 56,90 \\
\hline Média & $5,86 \pm 1,97$ & 33,56 & $5,61 \pm 1,80$ & 32,11 & $14,64 \pm 4,45$ & 30,37 & $9,61 \pm 5,48$ & 57,04 \\
\hline \multicolumn{9}{|l|}{ RCM } \\
\hline Direito & $1,99 \pm 0,72$ & 35,95 & $2,22 \pm 0,45$ & 20,16 & $1,85 \pm 0,69$ & 37,06 & $1,98 \pm 0,63$ & 32,53 \\
\hline Esquerdo & $2,19 \pm 0,93$ & 42,61 & $3,15^{\mathrm{C}} \pm 1,17$ & 37,14 & $1,89^{\mathrm{D}} \pm 0,24$ & 12,74 & $2,28 \pm 0,90$ & 38,89 \\
\hline Média & $2,09 \pm 0,82$ & 39,02 & $2,68 \pm 0,98$ & 36,41 & $1,87 \pm 0,50$ & 26,96 & $2,13 \pm 0,80$ & 37,35 \\
\hline
\end{tabular}

volume cortical esquerdo foram os únicos parâmetros, linear e volumétrico, a apresentarem diferença estatística entre suas médias, respectivamente. Já o grupo com idade de 6-18 meses quando comparado com o grupo com idade superior a 18 meses revelou que o comprimento medular esquerdo e a relação cortico-medular direita são os únicos parâmetros que não possuem suas médias diferentes entre os grupos.

Calculou-se o volume renal total por quilograma de peso corporal de cada rim de todas as fêmeas, para o rim esquerdo obteve-se uma média de $1,312 \pm 0,655 \mathrm{~cm}^{3}$ por kg de peso vivo, já para o rim direito a média foi de $1,133 \pm 0,467 \mathrm{~cm}^{3}$ por kg. A média da relação volume/peso corporal do rim esquerdo não difere estatisticamente do rim direito. Para os grupos com idade inferior a 6 meses, de 6-18 meses e com idade superior a 18 meses constatou-se, para o rim esquerdo, médias e desvio padrão de $1,771 \pm 0,802 \mathrm{~cm}^{3} / \mathrm{kg}$, $1,367 \pm 0,501 \mathrm{~cm}^{3} / \mathrm{kg}$, e $0,929 \pm 0,305 \mathrm{~cm}^{3} / \mathrm{kg}$, respectivamente; já para o rim direito obteve-se médias e desvio padrão de $1,603 \pm 0,458 \mathrm{~cm}^{3} \mathrm{~kg}, 0,962 \pm 0,220 \mathrm{~cm}^{3} / \mathrm{kg}$, e $0,861 \pm 0,254$ $\mathrm{cm}^{3} / \mathrm{kg}$. Por fim, observou-se que a média da relação volume/peso é estatisticamente diferente entre os dois rins somente para o grupo de fêmeas com idade de 6-18 meses.

A análise de correlação de Pearson, classificada de acordo com Santos (2007), quando realizada para todas as fêmeas, revelou que há correlação significativa (correlações moderada e forte positivas) entre peso e idade, e os parâmetros lineares (comprimento, largura e altura). Com relação aos parâmetros volumétricos observou-se que a idade e principalmente o peso apresentam correlação moderada positiva ou correlação forte positiva (significativas), com os volumes renal, cortical e medular, esquerdo e direito. É importante 
ressaltar que não houve correlação significativa entre a relação cortico-medular com outros parâmetros como idade, peso e volume, exceto para a relação cortico-medular direita e peso ( $r=-0,365$; $P$ 0,047), os outros valores encontrados mantiveram-se entre correlação ínfima e fracamente positiva, ou negativa (Quadro 3). Entretanto, quando se avaliaram os dados dos grupos isoladamente (fêmeas com idade inferior a 6 meses, de 6 a 18 meses e idade superior a 18 meses) o teste de correlação de Pearson não foi significativo $(\mathrm{P}>0,05)$. Considerando-se os parâmetros das fêmeas como um todo, para aqueles que apresentaram correlação signifi- cativa, foi realizada análise de regressão, obtendo-se a linha de melhor ajuste das variáveis (Quadro 4).

Nos planos de corte longitudinais (Fig.1) e transversais (Fig.2) realizados através das técnicas ultrassonográficas, observou-se a estrutura interna do parênquima renal e regiões vizinhas. Nestas imagens foi possível diferenciar a região cortical $(\mathrm{C})$ mais ecogênica quando comparado à região medular $(\mathrm{M})$. A pelve $(\mathrm{P})$ corresponde à região mais central formando o chamado complexo ecogênico central, composto pela pelve e gordura peripélvica. A cápsula (Cap) é, ocasionalmente, visualizada como um fino eco brilhan-

Quadro 3. Correlação de Pearson entre peso e idade com parâmetros métricos lineares e volumétricos das 30 fêmeas

\begin{tabular}{|c|c|c|c|c|c|c|c|c|c|c|}
\hline \multirow{2}{*}{\multicolumn{2}{|c|}{ Comprimento $(\mathrm{cm})$}} & \multicolumn{2}{|c|}{ Idade } & \multicolumn{2}{|c|}{ Peso } & & \multicolumn{2}{|c|}{ Idade } & \multicolumn{2}{|c|}{ Peso } \\
\hline & & $\mathrm{r}$ & P-value & $\mathrm{r}$ & P-value & & $\mathrm{r}$ & P-value & $\mathrm{r}$ & P-value \\
\hline & Direito & 0,778 & $<0,001$ & 0,764 & $<0,001$ & & & & & \\
\hline & Esquerdo & 0,809 & $<0,001$ & 0,809 & $<0,001$ & & & & & \\
\hline & Largura $(\mathrm{cm})$ & & & & & Volume Renal $\left(\mathrm{cm}^{3}\right)$ & & & & \\
\hline & Direito & 0,755 & $<0,001$ & 0,883 & $<0,001$ & Direito & 0,745 & $<0,001$ & 0,846 & $<0,001$ \\
\hline 牙 & $\begin{array}{l}\text { Esquerdo } \\
\text { Altura }(\mathrm{cm})\end{array}$ & 0,698 & $<0,001$ & 0,771 & $<0,001$ & $\begin{array}{l}\text { Esquerdo } \\
\text { Volume Cortical }\left(\mathrm{cm}^{3}\right)\end{array}$ & 0,725 & $<0,001$ & 0,775 & $<0,001$ \\
\hline & Direito & 0,700 & $<0,001$ & 0,811 & $<0,001$ & Direito & 0,653 & $<0,001$ & 0,738 & $<0,001$ \\
\hline & Esquerdo & 0,651 & $<0,001$ & 0,707 & $<0,001$ & $\begin{array}{l}\text { Esquerdo } \\
\text { Volume Medular }\left(\mathrm{cm}^{3}\right)\end{array}$ & 0,665 & $<0,001$ & 0,705 & $<0,001$ \\
\hline & Comprimento $(\mathrm{cm})$ & & & & & Direito & 0,752 & $<0,001$ & 0,861 & $<0,001$ \\
\hline & Direito & 0,679 & $<0,001$ & 0,756 & $<0,001$ & Esquerdo & 0,775 & $<0,001$ & 0,804 & $<0,001$ \\
\hline$\cong$ & Esquerdo & 0,752 & $<0,001$ & 0,730 & $<0,001$ & RCM & & & & \\
\hline & Largura $(\mathrm{cm})$ & & & & & Direito & $-0,201$ & 0,287 & $-0,220$ & 0,242 \\
\hline 5 & Direito & 0,745 & $<0,001$ & 0,879 & $<0,001$ & Esquerdo & $-0,346$ & 0,061 & $-0,365$ & 0,047 \\
\hline$\sum$ & $\begin{array}{l}\text { Esquerdo } \\
\text { Altura }(\mathrm{cm})\end{array}$ & 0,693 & $<0,001$ & 0,785 & $<0,001$ & & & & & \\
\hline & Direito & 0,717 & $<0,001$ & 0,780 & $<0,001$ & & & & & \\
\hline & Esquerdo & 0,735 & $<0,001$ & 0,804 & $<0,001$ & & & & & \\
\hline
\end{tabular}

Quadro 4. Análise de Regressão dos parâmetros métricos lineares e volumétricos com relação à idade e peso das $\mathbf{3 0}$ fêmeas

\begin{tabular}{|c|c|c|c|c|c|}
\hline & Comprimento $(\mathrm{cm})$ & Idade (meses) & & Peso & \\
\hline & & Relação & $\mathrm{R}^{2}$ & Relação & $\mathrm{R}^{2}$ \\
\hline & Direito & $\mathrm{CRD}=5,26+0,0216$ Idade & 0,606 & $\mathrm{CRD}=5,02+0,0261$ Peso & 0,583 \\
\hline & Esquerdo & $\mathrm{CRE}=5,38+0,0346$ Idade & 0,654 & $\mathrm{CRE}=4,97+0,0427$ Peso & 0,654 \\
\hline & Largura $(\mathrm{cm})$ & & & & \\
\hline & Direito & LRD $=2,49+0,0190$ Idade & 0,570 & LRD $=2,15+0,0275$ Peso & 0,780 \\
\hline 娄 & Esquerdo & LRE $=2,67+0,0150$ Idade & 0,487 & $\mathrm{LRE}=2,44+0,0204$ Peso & 0,594 \\
\hline$\sum_{1}$ & Altura $(\mathrm{cm})$ & & & & \\
\hline$\underline{\simeq}$ & Direito & $\mathrm{ARD}=2,38+0,0180$ Idade & 0,490 & $\mathrm{ARD}=2,06+0,0257$ Peso & 0,657 \\
\hline & Esquerdo & $\mathrm{ARE}=2,51+0,0147$ Idade & 0,424 & $\mathrm{ARE}=2,29+0,0198$ Peso & 0,500 \\
\hline & Volume $\left(\mathrm{cm}^{3}\right)$ & & & & \\
\hline & Direito & $\mathrm{VRD}=16,3+0,445$ Idade & 0,554 & VRD $=8,87+0,624$ Peso & 0,716 \\
\hline & Esquerdo & $\mathrm{VRE}=18,8+0,474$ Idade & 0,525 & $\mathrm{VRE}=12,0+0,626$ Peso & 0,600 \\
\hline 己 & Volume $\left(\mathrm{cm}^{3}\right)$ & & & & \\
\hline 宗 & Direito & $\mathrm{VCD}=10,9+0,270$ Idade & 0,426 & VCD $=6,45+0,377$ Peso & 0,545 \\
\hline ○ & Esquerdo & VCE $=13,2+0,292$ Idade & 0,442 & $\mathrm{VCE}=9,16+0,381$ Peso & 0,497 \\
\hline & Comprimento $(\mathrm{cm})$ & & & & \\
\hline & Direito & $\mathrm{CMD}=4,13+0,0140$ Idade & 0,461 & $\mathrm{CMD}=3,91+0,0193$ Peso & 0,571 \\
\hline & Esquerdo & $\mathrm{CME}=4,18+0,0265$ Idade & 0,565 & $\mathrm{CME}=3,89+0,0318$ Peso & 0,533 \\
\hline & Largura $(\mathrm{cm})$ & & & & \\
\hline$\stackrel{\Re}{\varangle}$ & Direito & $\mathrm{LMD}=1,68+0,0147$ Idade & 0,555 & LMD $=1,42+0,0213$ Peso & 0,773 \\
\hline S & Esquerdo & $\mathrm{LME}=1,71+0,0117$ Idade & 0,481 & $\mathrm{LME}=1,51+0,0164$ Peso & 0,616 \\
\hline 巠 & Altura $(\mathrm{cm})$ & & & & \\
\hline$\sum$ & Direito & $\mathrm{AMD}=1,47+0,0153$ Idade & 0,514 & $\mathrm{AMD}=1,24+0,0205$ Peso & 0,608 \\
\hline & Esquerdo & $\mathrm{AME}=1,48+0,0137$ Idade & 0,540 & $\mathrm{AME}=1,27+0,0185$ Peso & 0,647 \\
\hline & Volume $\left(\mathrm{cm}^{3}\right)$ & & & & \\
\hline & Direito & VMD $=5,38+0,174$ Idade & 0,565 & VMD $=2,42+0,247$ Peso & 0,741 \\
\hline & Esquerdo & VME $=5,54+0,183$ Idade & 0,601 & $\mathrm{VME}=2,84+0,244$ Peso & 0,705 \\
\hline
\end{tabular}




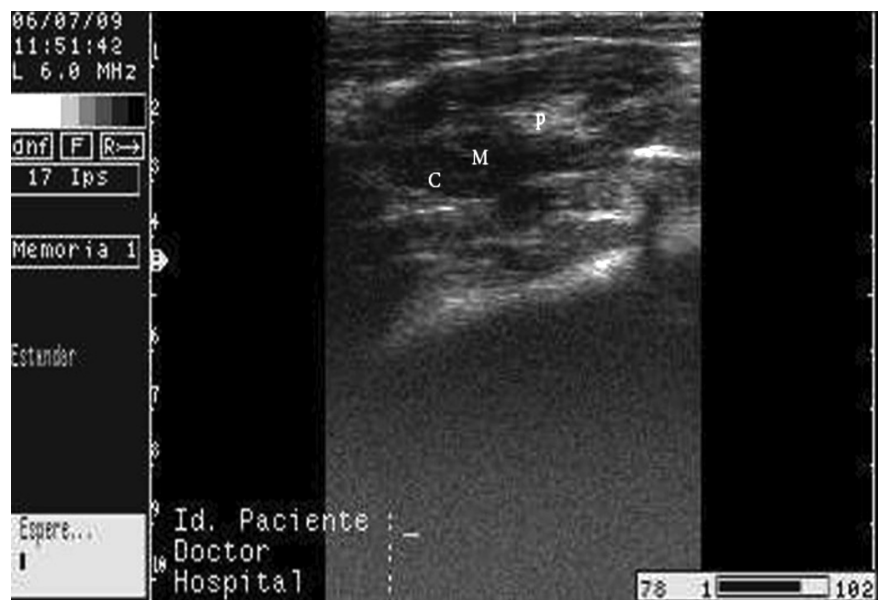

Fig.1. Ultrassonografia de plano de secção longitudinal do rim direito de animal com 3 meses e 12,5 kg.

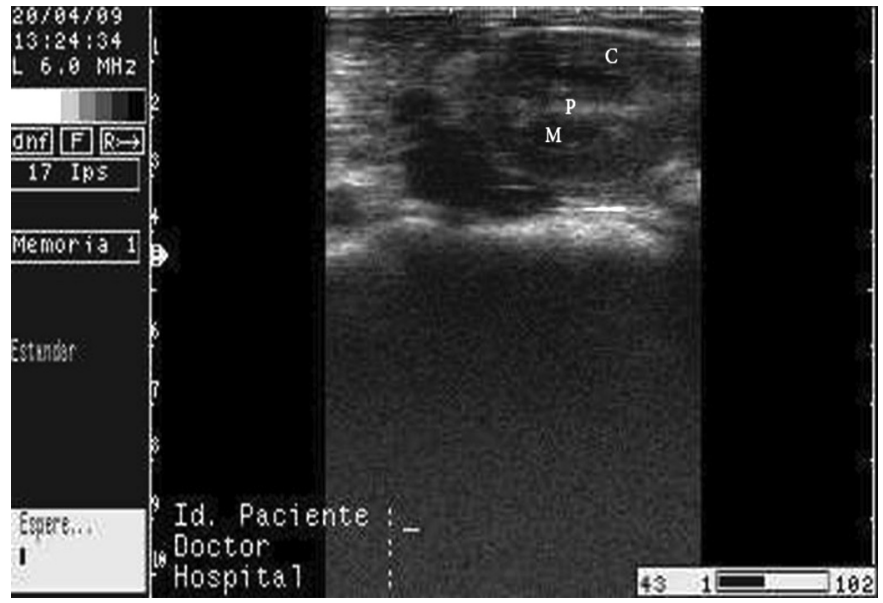

Fig.2. Ultrassonografia de plano de secção transversal do rim direito de animal com 20,2 meses e $34,7 \mathrm{~kg}$.

te, assim como o peritônio (Per). Em algumas imagens, em planos de secção longitudinais e transversais devido à posição cranial do rim direito e também ao fato deste encontrar-se sob o gradil costal, é possível a visualização de sombras acústicas (SA), causadas pelas costelas que podem dificultar a visualização.

\section{DISCUSSÃO}

A localização, posicionamento e disposição dos rins está de acordo com o descrito por Habel (1970), Evans \& Christensen (1993) e Dyce et al. (2004). Enfatizamos que, nos animais mais jovens estudados (menos de 1 ano), o pólo cranial dos dois rins encontra-se voltado para região cranial, e o pólo caudal voltado para região caudal, sendo que o rim esquerdo encontra-se caudal ao direito no mesmo plano horizontal. Com relação aos animais mais velhos (mais de 1 ano) foi possível observar que a porção caudal do rim direito pende ventralmente, mas, ainda assim, o rim ocupa a mesma posição. Já o rim esquerdo apresenta um posicionamento mais dinâmico, como mencionado pelos autores acima, este além de ter sua porção caudal pendente ventralmente, também se desloca ventralmente. A explicação para tal observação é dada devido ao fato de que quando há um crescimento corporal longitudinal dos animais, não ocorre um crescimento equivalente dos ureteres, os quais tracionam os rins de forma que a porção caudal destes penda ventralmente (P.P. Bombonato, informação verbal).

Assim como observado por Cunha (2005) e Kealy \& Mcallister (2005) durante a realização das imagens ultrassonográficas, contatou-se uma ligeira movimentação dos rins durante a respiração.

Em conformidade com a orientação de Felkai et. al. (1992), quando citado por Sampaio \& Araújo (2001), certos cuidados foram adotados para a obtenção das medidas ultrassonográficas. Nos planos, longitudinal e transversal, as dimensões de comprimento, largura e altura foram mensuradas em secções consideradas representativas da máxima medida renal, obtidas a partir do melhor posicionamento do transdutor. Para mensurar o comprimento renal, procurou-se utilizar imagens referentes aos planos de corte que apresentassem, quando possível, a visualização das extremidades, cranial e caudal, do rim. É importante ressaltar que, segundo Sampaio \& Araújo (2001), resultados demonstraram que os parâmetros lineares podem ser obtidos com segurança em qualquer plano anatômico de imagem.

A descrição da estrutura renal interna, assim como de seus padrões ecográficos observados no experimento, está de acordo com aquela relatada por Konde et al. (1984), Carvalho (2004), Cunha (2005), Kealy \& Mcallister (2005) e Piera (2005). Ressalta-se que todos estes autores procederam estudos ultrassonográficos renais em cães, realizando-se a comparação com estes devido à ausência de trabalhos sobre morfometria renal em ruminantes na literatura. Foi possível, ainda, identificar que a região cortical é mais ecogênica quando comparado à região medular em ambos os planos de corte, devido ao fato da região cortical possuir uma quantidade maior de células, enquanto a medula é composta pela maioria dos túbulos do sistema coletor, os quais possuem mais fluidos, tornando-a mais hipoecogênica. 0 conhecimento dos padrões normais é essencial para perceber padrões anormais que podem ser observados em uma enfermidade renal.

Com relação às variabilidades referentes às medidas lineares, ou seja, ao comprimento, largura e altura, renal e medular, tanto do rim esquerdo como do direito, para as fêmeas como um todo, foi possível constatar medidas consideradas controladas, mesmo com a variabilidade expressiva observada com relação à idade e peso, de acordo com os dados obtidos por Piera (2005). Quando se analisa os grupos individualmente, observa-se uma maior variabilidade, principalmente, com relação ao comprimento renal e medular, de ambos os rins, que tende a tornar-se controlada conforme os animais ficam mais velhos. Já o volume renal, volume cortical e volume medular, para os grupos isoladamente e como um todo, apresentaram variabilidade expressiva. Contraditoriamente a relação cortico-medular (VC/VM) encontrada apresentou baixa variabilidade. Os padrões anteriormente descritos também estão de acordo com os descritos por Piera (2005). 
Sampaio \& Araújo (2001) relataram que as médias das medidas lineares renais (comprimento, largura e espessura) entre os dois rins foram estatisticamente equivalentes, o que está de acordo com o observado para o grupo de animais com idade inferior a 6 meses e para o grupo de fêmeas como um todo, mas contraditoriamente a estes autores e em concordância com Barr et al. (1990), no presente trabalho encontrou-se que o comprimento renal, esquerdo e direito, não são estatisticamente equivalentes para os grupos com idade entre 6 e 18 meses e superior a 18 meses, além disso, para o último grupo o comprimento medular também apresentou média diferente entre os rins.

Avaliando-se o desenvolvimento renal por meio da comparação das médias obtidas nos diferentes grupos observou-se que no início ambos os rins apresentam-se com formato semelhante, entretanto, com o crescimento das fêmeas, estes começam a se diferenciar, acentuando-se a diferença conforme envelhecem, de forma que o rim esquerdo tende a tornar-se mais elipsóide, ou seja, mais comprido e afilado, enquanto o rim direito adquire um aspecto mais globoso.

Os parâmetros volumétricos, entre os dois rins, não diferiram entre si para os grupos de animais com idade inferior a 6 meses e superior a 18 meses, resultado que também foi constatado por Barr et al. (1990), Sampaio \& Araújo (2001) e Tello \& Requeséns (1995). Não obstante, para o grupo com idade entre 6 e 18 meses e considerando-se as fêmeas como um todo, obteve-se diferença estatística entre os rins para os parâmetros volume renal e volume cortical. Porém, vale ressaltar que assim como relatado por Barr et al. (1990), considerando-se os animais individualmente é comum que um rim tenha o volume maior do que o outro.

0 estudo da relação existente entre volume renal $\left(\mathrm{cm}^{3}\right)$ e peso corporal $(\mathrm{kg})$ dos animais realizado é uma forma de análise dos dados também abordada por Barr et al. (1990) e Sampaio \& Araújo (2001), mas para caprinos, não foram encontrados resultados na literatura para comparação.

Analisando-se os dados das fêmeas como um todo através da correlação de Pearson, observou-se que o peso corporal apresenta correlação significativa, direta e positiva com as medidas renais lineares, os seja, com o comprimento, largura e altura, em concordância com Barr et al. (1990), Tello \& Requeséns (1995), Sampaio \& Araújo (2001) e Cunha (2005). Assim, quanto maior o peso, maior o tamanho renal. Contraditoriamente ao relatado por Tello \& Requeséns (1995), no presente trabalho, a idade apresentou, predominantemente, moderada correlação com as mensurações referentes ao comprimento, largura e altura.

Ainda utilizando a técnica de regressão linear de Pearson para todas as fêmeas, em conformidade com o descrito por Barr et al. (1990), Felkai et. al. (1992), Tello \& Requeséns (1995), Sampaio \& Araújo (2001), Cunha (2005) e Piera (2005), constatou-se que o peso apresentou significativa correlação com o volume renal, volume cortical e volume medular. Com relação à idade, no presente trabalho, relatou-se moderada a forte correlação entre este parâmetro e os parâmetros volumétricos, em contrariedade com Tello \& Requeséns (1995) e Piera (2005). Assim como observado por Piera (2005), a análise da correlação de Pearson, demonstrou que a relação cortico-medular, tanto esquerda como direita, apresentou fraca e até mesmo ínfima correlação com outros parâmetros como peso, idade e volumes (renal, cortical e medular). Dessa forma podemos dizer que a relação cortico-medular não sofre interferência destas outras variáveis discutidas.

Da mesma forma que Barr et al. (1990), Tello \& Requeséns (1995), Sampaio \& Araújo (2001) e Cunha (2005), realizou-se análise de regressão dos dados e mensurou-se o grau de correlação entre os parâmetros que apresentaram correlação significativa considerando-se as fêmeas como um todo (peso e idade, com relação os parâmetros lineares e volumétricos), obtendo-se a reta de melhor ajuste e os valores de $\mathrm{R}^{2}$.

Com auxílio das mensurações morfométricas observou-se que a relação cortico-medular (RCM) apresentou pequena variabilidade diante da grande variação observada nos outros parâmetros tanto para os grupos individualmente como para todas as fêmeas, similarmente ao que foi constatado por Piera (2005).

Por fim, concordamos com, Tello \& Requeséns (1995) e Sampaio \& Araújo (2001), que a determinação precoces das variações do volume renal pode prover ao clínico informações rápidas e valiosas das afecções patológicas que podem acometer os rins, mesmo antes da aparição dos sinais clínicos. A possibilidade de detecção precoce de uma nefropatia assegura que a terapia e o manejo adotados terão maiores possibilidades de êxito. Além disso, a quantidade de informações obtidas por meio do exame ultrassonográfico renal revelou a grande importância deste exame complementar, mesmo que, certas vezes, o tamanho normal dos rins não exclua a possibilidade de doença renal.

Os dados referentes às medidas morfométricas lineares e volumétricas renais por meio da ultrassonografia, ainda não foram descritos na literatura para caprinos (Capra hircus), sendo provavelmente os primeiros a serem relatados nesta espécie. Em virtude deste fato muitas comparações foram efetuadas entre caprinos e cães, espécie que possui um estudo mais aprofundado nesta área. Apesar de tratar-se de duas espécies diferentes, vários aspectos comparados entre o presente trabalho e outros autores mostraram resultados semelhantes.

\section{CONCLUSÕES}

De acordo com os dados obtidos nesse estudo e suas interpretações com base na literatura, pode-se concluir que os intervalos normais dos parâmetros morfométricos renais lineares e volumétricos de fêmeas caprinas da raça Saanen são como segue: 


\begin{tabular}{|c|c|c|c|c|}
\hline \multicolumn{5}{|c|}{ LINEARES } \\
\hline & $<6$ & 6 a 18 & $>18$ & Média \\
\hline & Meses & Meses & Meses & \\
\hline \multicolumn{5}{|c|}{ Comprimento $(\mathrm{cm})$} \\
\hline Direito & $5,32 \pm 0,38$ & $5,29 \pm 0,13$ & $6,35 \pm 0,50$ & $5,8 \pm 0,65$ \\
\hline Esquerdo & $5,14 \pm 0,34$ & $5,83 \pm 0,40$ & $7,18 \pm 0,49$ & $6,2 \pm 1,01$ \\
\hline Média & $5,20 \pm 0,36$ & $5,56 \pm 0,40$ & $6,77 \pm 0,64$ & $6,0 \pm 0,87$ \\
\hline \multicolumn{5}{|c|}{ Largura $(\mathrm{cm})$} \\
\hline$\Rightarrow$ Direito & $2,42 \pm 0,21$ & $2,63 \pm 0,32$ & $3,50 \pm 0,40$ & $2,9 \pm 0,60$ \\
\hline$\sum_{x=1}^{K}$ Esquerdo & $2,60 \pm 0,33$ & $2,86 \pm 0,22$ & $3,42 \pm 0,42$ & $3.0 \pm 0,51$ \\
\hline Média & $2,50 \pm 0,29$ & $2,27 \pm 0,29$ & $3,46 \pm 0,40$ & $3,0 \pm 0,55$ \\
\hline \multicolumn{5}{|c|}{ Altura (cm) } \\
\hline Direito & $2,43 \pm 0,23$ & $2,28 \pm 0,35$ & $3,35 \pm 0,43$ & $2,80 \pm 0,61$ \\
\hline Esquerdo & $2,48 \pm 0,26$ & $2,63 \pm 0,42$ & $3,25 \pm 0,49$ & $2,28 \pm 0,53$ \\
\hline Média & $2,50 \pm 0,24$ & $2,46 \pm 0,41$ & $3,30 \pm 0,46$ & $2,80 \pm 0,57$ \\
\hline \multicolumn{5}{|c|}{ Comprimento $(\mathrm{cm})$} \\
\hline Direito & $4,08 \pm 0,42$ & $4,23 \pm 0,22$ & $4,87 \pm 0,31$ & $4,5 \pm 0,49$ \\
\hline Esquerdo & $4,07 \pm 0,55$ & $4,41 \pm 0,45$ & $5,55 \pm 0,46$ & $4,8 \pm 0,83$ \\
\hline Média & $4,10 \pm 0,47$ & $4,32 \pm 0,35$ & $5,21 \pm 0,52$ & $4,6 \pm 0,70$ \\
\hline \multicolumn{5}{|c|}{$\dddot{2}$ Largura $(\mathrm{cm})$} \\
\hline Direito & $1,70 \pm 0,20$ & $1,72 \pm 0,26$ & $2,44 \pm 0,36$ & $2,0 \pm 0,46$ \\
\hline Esquerdo & $1,72 \pm 0,27$ & $1,76 \pm 0,30$ & $2,29 \pm 0,32$ & $2,0 \pm 0,40$ \\
\hline$\sum$ Média & $1,70 \pm 0,23$ & $1,74 \pm 0,27$ & $2,36 \pm 0,34$ & $2,0 \pm 0,43$ \\
\hline \multicolumn{5}{|c|}{ Altura (cm) } \\
\hline Direito & $1,56 \pm 0,21$ & $1,37 \pm 0,20$ & $2,28 \pm 0,40$ & $1,80 \pm 0,50$ \\
\hline Esquerdo & $1,56 \pm 0,24$ & $1,41 \pm 0,16$ & $2,19 \pm 0,34$ & $1,80 \pm 0,44$ \\
\hline Média & $1,60 \pm 0,22$ & $1,39 \pm 0,18$ & $2,23 \pm 0,37$ & $1,80 \pm 0,47$ \\
\hline \multicolumn{5}{|c|}{ VOLUMÉTRICOS } \\
\hline & $<6$ & 6 a 18 & $>18$ & Média \\
\hline & Meses & Meses & Meses & \\
\hline \multicolumn{5}{|c|}{ Volume Renal $\left(\mathrm{cm}^{3}\right)$} \\
\hline Direito & $16,48 \pm 3,40$ & $16,82 \pm 4,16$ & $39,68 \pm 11,72$ & $26,21 \pm 13,87$ \\
\hline Esquerdo & $17,56 \pm 4,63$ & $23,15 \pm 5,80$ & $42,77 \pm 14,57$ & $29,79 \pm 15,20$ \\
\hline Média & $17,02 \pm 3,99$ & $19,99 \pm 5,86$ & $41,23 \pm 13,05$ & $28,20 \pm 14,76$ \\
\hline \multicolumn{5}{|c|}{ Volume Cortical $(\mathrm{cm} 3)$} \\
\hline Direito & $10,70 \pm 2,42$ & $11,54 \pm 3,07$ & $25,21 \pm 9,94$ & $17,18 \pm 9,62$ \\
\hline Esquerdo & $11,62 \pm 3,45$ & $17,21 \pm 4,94$ & $27,97 \pm 10,32$ & $20,01 \pm 10,19$ \\
\hline Média & $11,16 \pm 2,94$ & $14,37 \pm 4,92$ & $26,59 \pm 10,03$ & $18,60 \pm 10,09$ \\
\hline \multicolumn{5}{|c|}{$\begin{array}{l}\text { Volume Medular } \\
(\mathrm{cm} 3)\end{array}$} \\
\hline Direito & $5,78 \pm 1,68$ & $5,28 \pm 1,37$ & $14,48 \pm 4,56$ & $9,43 \pm 5,39$ \\
\hline Esquerdo & $5,94 \pm 2,31$ & $5,95 \pm 2,21$ & $14,80 \pm 4,51$ & $9,78 \pm 5,47$ \\
\hline Média & $5,86 \pm 1,97$ & $5,61 \pm 1,80$ & $14,64 \pm 4,45$ & $9,61 \pm 5,48$ \\
\hline \multicolumn{5}{|l|}{ RCM } \\
\hline Direito & $1,99 \pm 0,72$ & $2,22 \pm 0,45$ & $1,85 \pm 0,69$ & $1,98 \pm 0,63$ \\
\hline Esquerdo & $2,19 \pm 0,93$ & $3,15 \pm 1,17$ & $1,89 \pm 0,24$ & $2,28 \pm 0,90$ \\
\hline Média & $2,09 \pm 0,82$ & $2,68 \pm 0,98$ & $1,87 \pm 0,50$ & $2,13 \pm 0,80$ \\
\hline
\end{tabular}

Além disso, também chegamos às seguintes conclusões: Para os animais com idade inferior a 6 meses

- 0 comprimento renal esquerdo $(5,14 \pm 0,34)$ e direito $(5,32 \pm 0,38)$, a largura renal esquerda $(2,60 \pm 0,33)$ e direita $(2,42 \pm 0,21)$ a altura renal esquerda $(2,48 \pm$ $0,26)$ e direita $(2,43 \pm 0,23)$, o comprimento medular esquerdo $(4,07 \pm 0,55)$ e direito $(4,08 \pm 0,42)$, a largura medular esquerda $(1,72 \pm 0,27)$ e direita $(1,70$ $\pm 0,20)$, e, a altura medular esquerda $(1,56 \pm 0,24)$ e direita $(1,56 \pm 0,21)$ apresentam suas médias estatisticamente equivalentes entre os dois rins;

- 0 volume renal esquerdo $(17,56 \pm 4,63)$ e direito $(16,48 \pm 3,40)$, o volume cortical esquerdo $(11,62 \pm$ $3,45)$ e direito $(10,70 \pm 2,42)$, o volume medular esquerdo $(5,94 \pm 2,31)$ e direito $(5,78 \pm 1,68)$, e, a relação cortico-medular esquerda $(2,19 \pm 0,93)$ e direita $(1,99 \pm 0,72)$ apresentam suas médias estatisticamente equivalentes entre os rins.

\section{Para os animais com idade de 6 a 18 meses}

- A largura renal esquerda $(2,86 \pm 0,22)$ e direita $(2,63$ $\pm 0,32)$, a altura renal esquerda $(2,63 \pm 0,42)$ e direita $(2,28 \pm 0,35)$, o comprimento medular esquerdo $(4,41$ $\pm 0,45)$ e direito $(4,23 \pm 0,22)$, a largura medular esquerda $(1,76 \pm 0,30)$ e direita $(1,72 \pm 0,26)$, e, a altura medular esquerda $(1,41 \pm 0,16)$ e direita $(1,37 \pm 0,20)$ apresentam suas médias estatisticamente equivalentes entre os dois rins. No entanto, as médias do comprimento renal esquerdo $(5,83 \pm 0,40)$ e direito $(5,29$ $\pm 0,13)$ são estatisticamente diferentes;

- 0 volume medular esquerdo $(5,95 \pm 2,21)$ e direito $(5,28 \pm 1,37)$, e, a relação cortico-medular esquerda $(2,19 \pm 0,93)$ e direita $(2,22 \pm 0,45)$ apresentam suas médias estatisticamente equivalentes entre os rins. Já o volume renal esquerdo $(23,15 \pm 5,80)$ e direito $(16,82 \pm 4,16)$, e, o volume cortical esquerdo $(17,21 \pm$ $4,94)$ e direito $(11,54 \pm 3,07)$ possuem diferença estatística entre suas médias.

\section{Para os animais com idade superior a 18 meses}

- A largura renal esquerda $(3,42 \pm 0,42)$ e direita $(3,50$ $\pm 0,40)$, a altura renal esquerda $(3,25 \pm 0,49)$ e direita $(3,35 \pm 0,43)$, a largura medular esquerda $(2,29 \pm$ $0,32)$ e direita $(2,44 \pm 0,36)$, e, a altura medular esquerda $(2,19 \pm 0,34)$ e direita $(2,28 \pm 0,40)$ apresentam suas médias estatisticamente equivalentes entre os dois rins. Enquanto as médias do comprimento renal esquerdo $(7,18 \pm 0,49)$ e direito $(6,35 \pm 0,50)$, e, do comprimento medular esquerdo $(5,55 \pm 0,46)$ e direito $(4,87 \pm 0,31)$ são estatisticamente diferentes;

- 0 volume renal esquerdo $(42,77 \pm 14,57)$ e direito $(39,68 \pm 11,72)$, o volume cortical esquerdo $(27,97 \pm$ $10,32)$ e direito $(25,21 \pm 9,94)$, o volume medular esquerdo $(14,80 \pm 4,51)$ e direito $(14,48 \pm 4,56)$, e, a relação cortico-medular esquerda $(1,89 \pm 0,24)$ e direita $(1,85 \pm 0,69)$ apresentam suas médias estatisticamente equivalentes entre os rins.

\section{Para todos os animais}

- A relação cortico-medular apresenta pouca variabilidade frente à grande variação apresentada pelos volumes renal, cortical e medular, peso e idade;

- A relação cortico-medular não sofre interferência pelo peso, idade ou volumes (renal, cortical e medular) renais;

- Os parâmetros lineares, comprimento, largura e altura renais, apresentam correlação forte, direta e positiva com o peso. Já com a idade a correlação apresentou-se moderada, direta e positiva;

- Os parâmetros volumétricos, volume renal, volume cortical e volume medular, apresentam correlação forte, direta e positiva com o peso. Com a idade esta correlação apresenta-se moderada, direta e positiva;

- 0 comprimento, largura e altura, tanto renal como medular, apresentaram médias estatisticamente equivalentes entre o rim esquerdo e o direito. Com relação aos parâmetros volumétricos, somente o volume medular e a relação cortico-medular apresentaram médias estatística equivalente entre os dois rins; 
- Entre o grupo de fêmeas com idade inferior a 6 meses e o com idade entre 6 a 18 meses o comprimento renal esquerdo e o volume cortical esquerdo apresentam diferença estatística entre suas médias. Comparando-se este último grupo e o de fêmeas com idade superior a 18 meses, os únicos parâmetros equivalentes entre as médias dos grupos são o comprimento medular esquerdo e a relação cortico-medular direita.

Agradecimentos.- Ao Conselho Nacional de Desenvolvimento Científico e Tecnológico (CNPq), pelo auxílio financeiro.

\section{REFERÊNCIAS}

Barr E.J. 1990. Evaluation of ultrasound as a method of assessing renal size in the dog. J. Small Anim. Pract. 31:174-179.

Barr F.J., Holt P.E. \& Gibbs C. 1990. Ultrasonographic measurement of normal renal parameters. J. Small Anim. Pract. 31:180-184.

Bakker J., Olree M., Katte R., Lange E.E., Moons K.G.M., Beutler J.J. \& Beek F.J.A. 1999. Renal Volume measurements: Accuracy and Repeatability of US compared with That of MR Imaging. Radiology 211:623-628.

Berquó E.S., Souza J.M.P. \& Gotlieb S.L.D. 2002. Análise descritiva de variáveis quantitativas: medidas de posição, de variabilidade, de assimetria e de achatamento; noções sobre correlação e regressão, p.63-113. In: Ibid. (Eds), Bioestatística. 2ํㅡㄹ ed. EPU, São Paulo. 350p.

Carvalho C.F. 2004. Sistema urinário: rins, ureteres, bexiga urinária e uretra, p.111-146. In: Ibid. (Eds), Ultra-sonografia em Pequenos Animais. Roca Ltda, São Paulo. 365p.

Coles E.H. 1986. Kidney function, p.171-202. In: Ibid. (Eds), Veterinary Clinical Pathology. $4^{\text {th }}$ ed. Saunders Company, Philadelphia. 486p.

Cunha L.M.F. 2005. Correlação entre peso corporal e volume renal por medidas lineares ultrassonográficas em cães da raça Dachshund. Dissertação de Mestrado em Ciências Veterinárias, Faculdade de Medicina Veterinária, Universidade Federal de Uberlândia, Uberlândia, MG. 38p.

Dibartola S.P. 1997. Abordagem clínica e avaliação laboratorial da afecção renal, p.2355-2373. In: Ettinger S.J. \& Feldman E.C. (Eds), Tratado de Medicina Interna Veterinária: doenças do cão e do gato. Vol.2. 4⿳⺈ ed. Manole, São Paulo.
Dyce K.M., Sack W.O. \& Wensing C.J.G. 2004. Tratado de Anatomia Veterinária. $3^{\underline{a}}$ ed. Elsevier, Rio de Janeiro. 813p.

Evans H.E. \& Christensen G.C. 1993. The urogenital system, p.494-558. In: Ibid. (Eds), Miller's Anatomy of the Dog. $3^{\underline{a}}$ ed. Saunders Company, Philadelphia. 1113p.

Felkai C. S., Vörös K., Vrabély T. \& Karsai F. 1992. Ultrasonographic determination of renal volume in the dog. Vet. Radiol. Ultras. 33:292-296.

Gilka O. H., Hausken T., Berstad A. \& Odegaad S. 1999. Measurements of organ volume by ultrasonography. Proc. Inst. Mechanical Engineers 213:247-259.

Habel R.E. 1967. El abdomen: riñón, útero y ovario, p.33-65. In: Ibid. (Ed.), Guide to the Dissection of Domestic Ruminants. $2^{\mathrm{a}}$ ed. Acriba, Zaragoza. 198p.

Kaneko J.J., Harvey J.W. \& Bruss M.L. 1997. Kidney function, p.441-484. In: Finco D.R. (Ed.), Clinical Biochemistry of Domestic Animal. $4 \stackrel{\text { a }}{\text { ed. Acade- }}$ mic Press, San Diego. 932p.

Kealy J.K. \& McAllister H. 2005. The abdomen, p.21-171. In : Ibid. (Eds), Diagnostic Radiology Ultrasonography of the Dog and Cat. 4th ed. Elsevier Saunders, Philadelphia. 512p.

Konde L.J. 1989. Renal sonography. Sem. Vet. Med. Surgery, Small Anim. 4:32-43.

Konde L.J., Wrigley R.H., Park R.D. \& Lebel J.L. 1984. Ultrassonographic anatomy of the normal canine kidney. Vet. Radiol. 25:173-178.

Nyland T.G., Kantrowitz B.M., Fisher P., Olander H.J. \& Ornof W.J. 1989. Ultrasonic determination of kidney volume in the dog. Vet. Radiol. 30:174180.

Piera R.E. 2005. Mensuração ultra-sonográfica da relação cortico-medular renal em cães. Dissertação de Mestrado em Ciências Veterinárias, Faculdade de Medicina Veterinária e Zootecnia, Universidade de São Paulo, São Paulo, SP. 74p.

Sampaio K.M.O.R., Araújo R.B. 2002. Ultrassonografia de características lineares e estimativas do volume de rins de cães. Arq. Bras. Med. Vet. Zootec. 54:248-254.

Santos C. 2007. Estatística Descritiva: Manual de auto-aprendizagem. Silabo, Lisboa. 264p.

Tello L. \& Requeséns J. 1995. Volumetria ecográfica renal y su correlación com el peso corporal y edad em caninos adultos. Av. Cienc. Vet. 10:117123. 